\title{
On audiovisual spatial synergy: The fragility of the phenomenon
}

\author{
SALVADOR SOTO-FARACO \\ Universitat de Barcelona, Barcelona, Spain \\ and \\ SHARON MOREIN-ZAMIR and ALAN KINGSTONE \\ University of British Columbia, Vancouver, British Columbia, Canada
}

\begin{abstract}
Recent literature has highlighted the importance and ubiquity of cross-modal links in spatial attention, whereby shifts in attention in one modality often induce corresponding shifts in other modalities. We attempted to provide further evidence for the case of audiovisual links during sustained endogenous attention by addressing several potential methodological confounds in previous demonstrations. However, we failed repeatedly to reproduce the phenomenon of spatial synergies between auditory and visual attention, found by Driver and Spence (1994) and frequently cited to support the automatic nature of cross-modal attention links. We discuss the results in light of recent evidence about cross-modal spatial links during sustained attention and support the idea that such links can weaken or even disappear under certain circumstances, such as during periods of sustained attention. The implication is that individuals can select inputs from different modalities from different locations more easily than previously had been thought.
\end{abstract}

Mechanisms of selective attention must often operate in the context of multiple inputs coming from different sensory modalities. Although traditional research in attention has often restricted the scope of its enquiry to one sensory modality at a time, recent years have seen an important revival of interest regarding the question of how selection mechanisms are coordinated across different sensory modalities in the human brain. This research has provided a great deal of evidence for the existence of extensive cross-modal links in spatial attention (see Driver $\&$ Spence, 1998, 2004, for reviews). However, important questions about the nature of such cross-modal links still remain open. Here, we focus on the consequences of sustaining voluntary (endogenous) spatial attention in one modality (vision) for the distribution of spatial attention in another modality (audition).

\section{Cross-Modal Links in Endogenous Spatial Attention}

Many researchers now agree that orienting voluntary attention (overtly and also covertly) to a particular spatial location in search for a target in one sensory modal-

This work was supported by a postdoctoral award from the Killam Trust to S.S.-F., by a predoctoral scholarship from the University of British Columbia to S.M.-Z., and by grants from the National Science and Engineering Research Council of Canada, the Human Frontier Science Programme, and the Michael Smith Foundation for Medical Sciences to A.K. We thank Nuria Sebastián-Gallés, Albert Costa, Mari Ries Jones, and three anonymous reviewers for comments on a previous draft of this article. Correspondence should be addressed to S. Soto-Faraco, Departament de Psicologia Bàsica, Pg. de la Vall d'Hebrón, 171, 08035 Barcelona, Spain (e-mail: ssoto@ub.es). ity implies a corresponding shift of attention in other sensory modalities (e.g., Butchel \& Butter, 1988; Eimer, 1999; Hillyard, Simpson, Woods, Van Voorhis, \& Münte, 1984; Lloyd, Merat, McGlone, \& Spence, 2003; Spence \& Driver, 1996; Spence, Pavani, \& Driver, 2000; TederSälejärvi, Münte, Sperlich, \& Hillyard, 1999). This cross-modal coupling implies that when spatial attention shifts to a particular location in one modality, attention in other sensory modalities is to some extent summoned to that same location, which makes it difficult to select targets in different modalities appearing at different locations. For example, Spence and Driver (1996) used a spatialcuing paradigm in a study regarding audiovisual links in endogenous attention. In this paradigm, the observer is warned about the likely location of the upcoming target through a probabilistic manipulation involving a central cue (i.e., an arrowhead) or by making one target location more probable than the other (see Posner, 1980, for an earlier application of this paradigm). Spence and Driver (1996) were able to show that targets in a secondary (less frequent) modality benefited from being presented at the location where targets in the primary (more frequent) modality were expected, even though targets in the secondary modality were twice as likely to appear at the opposite location (see also Lloyd et al., 2003; Spence, Pavani, \& Driver, 2000). Their data strongly suggested that a voluntary shift in visual or auditory attention automatically induces a corresponding shift in the other modality (see Eimer, 1999, for similar findings, using event related potentials).

However, in these experiments, attention is often redirected to a new location from one trial to the next and 
may not be informative as to whether cross-modal links are sustained over time. Note that this is true even in experiments in which target location was fixed in each modality throughout a whole block of trials and central spatially informative cues were not used (e.g., Eimer, 1999; Spence \& Driver, 1996). A new attention shift may still have been initiated at each trial, because central fixation was reinforced by warning signals and eye tracking during the intertrial period. As was suggested by Sturm et al. (1999), even in the absence of external cuing, the level of alertness can be modulated in a top-down mode in self-initiated preparation for an upcoming target.

Testing whether spatial synergies across modalities outlive the initial attention shift (orienting) has important implications for human performance in a wide variety of situations (e.g., driving a car). That is, one important question in this field is to what degree this cross-modal coupling persists during sustained attentional effort (see Pashler, 1997, p. 90). Regarding this issue, several authors have pointed out an essential difference between the mechanisms leading to a shift in attention from one location to another and the consequences that attention has, once allocated to a particular location (LaBerge, 1995). Eimer and Van Velzen (2002) have recently argued that phasic attention shifts and tonic attention states (where attention must be sustained over a prolonged period of time) can have different consequences, specifically in terms of cross-modal attention (see also Eimer, 1996, for a similar argument regarding visual attention). For example, shifts in tactile attention can be observed as a consequence of phasic shifts of visual attention (Eimer \& Van Velzen, 2002), exactly as in the case of visual and auditory experiments. Yet several studies have now shown that tactile attention does not persist for long at the location where visual or auditory attention has been committed. These results have prompted some authors to claim that tactile attention is decoupled from vision and from audition during sustained attention states (Eimer \& Driver, 2000; Eimer, van Velzen, \& Driver, 2002; but see Hötting, Rösler, \& Röder, 2002).

\section{Are Vision and Audition Coupled During Sustained Attention?}

The results of early studies suggested that people tend to orient visually to the source of the sounds to which they are trying to listen, as if orienting visual attention would result in better listening (e.g., Gopher, 1973). This is perhaps not surprising, since in naturalistic environments, auditory information may be accompanied by redundant visual cues (see, e.g., Grant \& Seitz, 1998, 2000; Reisberg, 1978; Sumby \& Pollack, 1954). Following up on these early observations, Reisberg, Scheiber, and Potemken (1981) measured delayed recall of words belonging to one of two lists presented simultaneously from different locations. Recall accuracy was worse when participants looked at the source of irrelevant words, as compared with looking either at the source of relevant words or away from both relevant and irrelevant sound sources. This result indicates that looking at the source of distractor sounds interferes with the processing of relevant sounds presented at another location. However, Reisberg et al.'s results were somewhat puzzling, because they also seemed to indicate that looking at a location with no auditory stimuli (i.e., away from the relevant and the irrelevant sounds) had the same benefit to performance as looking at the location of relevant sounds. Indeed, a later study by Wolters and Schiano (1989) failed twice to replicate this pattern of results, since they did not find a cost of looking at the source of irrelevant sounds, as compared with looking toward the relevant sound source or to a location where no sounds were presented. There are several potential reasons for the failure to find a reliable cross-modal synergy triggered by gaze direction. First, both in Reisberg et al.'s and in Wolters and Schiano's studies, the sound sources were arranged vertically, a spatial dimension in which auditory localization is relatively poor (e.g., Blauert, 1997). Second, the recall task may not have been a sufficiently sensitive measure of attention. That is, forgetting during the interval between list presentation and the memory test may have abolished any attentional effects. Yet the most important concern is that the direction of gaze (overt orienting) may not be a reliable indicator of where visual attention is being allocated (e.g., Sperling, 1960). Thus, even if participants directed their gaze away from the relevant sound source, it does not follow that covert visual attention was summoned from the location of the relevant sound source (note, for example, that Spence \& Driver's, 1996, effects, described earlier, were found in the absence of eye movements).

Driver and Spence (1994) offered some of the most convincing evidence to date about the existence of links across audition and vision during sustained attention. In their study (Driver \& Spence, 1994, Experiment 2), participants performed an immediate recall task on word triplets presented aurally from one location (placed to the left or to the right) while attempting to ignore distractor words presented simultaneously from another location (placed to the right or to the left, respectively). Critically, at the same time, the participants were also asked to look at a visual display that was placed at the location of the relevant auditory sequence or at the location of the irrelevant auditory sequence (although the participants' heads were fixed, they could move their eyes to the left or the right). In the active condition, the visual display contained a rapid sequence of keyboard symbols, and the participants stopped the word recall task and responded every time they saw a + sign. In the passive condition, the visual sequence consisted of the letters $\mathrm{Z}$ and $\mathrm{O}$, alternating rapidly, and the participants simply looked at the display. Driver and Spence (1994, Experiment 2) found no effect of passively looking in the direction of (as opposed to away from) the relevant sounds, confirming the results obtained by Wolters and Schiano (1989). However, in the active-looking condition, they observed an advantage of visually attending to the source of relevant sounds, as opposed to visually attending to the location of irrelevant sounds. This result 
suggests that it is not possible to focus visual and auditory attention effectively to opposite locations in space, thus supporting the existence of cross-modal links during sustained endogenous attentional states. In a subsequent experiment, Driver and Spence (1994) found not only that this effect was due to the cost of visually attending to the location of the auditory distractors, but also that there was a net benefit of attending to the source of relevant sounds. This finding has very important implications. Not only does it suggest that auditory and visual attention are linked across space, but also it implies that this synergy persists over time and cannot be overcome even if detrimental to performance. This places a strong limitation on human performance; for example, in the case in which one is driving when using a mobile phone, even a hand-free headset model, there should be a detriment in performance.

These findings also pose some important constraints on theories of attention. Driver and Spence's (1994) results contrast with the predictions of independent-channel theories, which specify that the use of different sensory modalities should allow interference-free performance (e.g., Duncan, Martens, \& Ward, 1997; Wickens, 1984). They also complement those of a later study by Spence and Driver (1996), in which cross-modal links were shown in a trial-by-trial (blocked cuing) experiment (i.e., one putatively involving phasic, rather than sustained, attention shifts). Finally, Driver and Spence's (1994) audiovisual data also contrast with recent findings suggesting that touch can be decoupled from audition and from vision when attention must be sustained over time (Eimer \& Driver, 2000; Eimer et al., 2002), perhaps suggesting that different modality combinations can give rise to different outcomes (see also Soto-Faraco et al., 2002).

The basic paradigm introduced by Driver and Spence (1994) has been used in slightly modified forms on at least two other occasions (Spence, Ranson, \& Driver, 2000; Spence \& Read, 2003). On both occasions, the paradigm has included visual active and passive attentional conditions during a spatially selective auditory-shadowing task. It is perhaps important to note that neither of these studies included a direct replication of the condition of spatial coincidence between the relevant auditory message and the direction of visual attention (a condition that should lead to a beneficial effect on shadowing), and they measured only the detrimental effect of presenting irrelevant sounds at the location of visual attention. However, these studies should be interpreted with caution. As in Driver and Spence's (1994) original study, the visual stimuli were different in the active and the passive conditions. In the active condition, participants were exposed to a more attention-grabbing visual display than in the passive condition, and thus, the visual input could have a potentially different contribution in terms of reflexively or involuntarily attracting attention in the two conditions (see below).

\section{Scope of the Present Study}

The initial goal of the present study was to explore further the nature of audiovisual links in sustained en- dogenous spatial attention. To this end, we attempted to make use of the significant, albeit small, effect ${ }^{1}$ (about $7 \%$ ) of directing visual attention to the location of auditory attention found by Driver and Spence (1994). There are several concerns (some of them noted above) regarding Driver and Spence's (1994) paradigm and its successive applications (Spence, Ranson, \& Driver, 2000; Spence \& Read, 2003). First, the paradigm does not provide a measure of visual attention in the passive condition, and it is therefore difficult to assess the effectiveness of the manipulation of visual attention. One could argue that auditory shadowing, being worse in the active condition than in the passive-looking condition, provides sufficient evidence that visual attention was engaged to a larger extent in the latter. However, the argument becomes circular, because the differences in auditoryshadowing performance are explained by the different levels of attentional engagement in the visual task. This issue becomes critical because in Driver and Spence's (1994) experiments, as well as in subsequent experiments (Spence, Ranson, \& Driver, 2000; Spence \& Read, 2003), the displays used in the passive-looking condition were physically different from the displays used in the active condition. The visual displays in the active condition contained more variation and a higher degree of uncertainty than the visual displays in the passive condition did. These differences raise the concern that the visual displays in the active condition were more effective in engaging reflexive (i.e., involuntary) attention than the displays used in the passive-looking condition, thus causing the differences in auditory-shadowing performance. An additional concern is the nature of the auditory task in Driver and Spence (1994; also in Spence, Ranson, \& Driver, 2000; Spence \& Read, 2003). In this paradigm, a sustained attention state was achieved by presenting the targets from the same side throughout a block (both modalities from one side or each modality from a different side). However, because the presentation of the auditory words was in the form of triplets separated by several seconds in order to allow responding, it is possible that spatial attention may have not been distributed equally over time. Instead, this situation may have led to the reinitiation of (phasic) shifts in auditory attention prior to the presentation of each triplet, thus approaching the conditions of a trial-by-trial design (as in Spence \& Driver, 1996). Because of these concerns and the important implications of Driver and Spence's (1994) results, we sought to replicate and extend the initial paradigm.

We began with a manipulation with which we tried to reproduce Driver and Spence's (1994) results but in which (1) the visual displays used in the passive and the active conditions were physically identical, (2) the attention state was more uniformly sustained across time, thanks to the use of a continuous shadowing task (as opposed to a triplet-by-triplet shadowing task), and (3) the degree of visual attention could be monitored in each condition by introducing different levels of attentional demands (rather than a no-attention vs. attention comparison). What we found, however, was that the original 
results did not replicate in the different experiments we conducted, each one using a methodology increasingly similar to that in the original Driver and Spence (1994) study (until the final experiment, where the conditions were matched in almost every possible detail). Together, these data point to the interesting and important implication that individuals can select inputs from different modalities coming from different locations more easily than has previously been thought.

\section{EXPERIMENT 1}

The aim of the first experiment was to reproduce the linked attention effect, whereby auditory-shadowing performance improves when visual attention is directed to the location of the relevant sounds, versus when attention is directed to a different location containing irrelevant sounds. Two sequences of auditory words were presented simultaneously, one originating from the participant's right and one originating from his/her left. The participants shadowed one of the two sequences while performing a visual detection task. We varied the attentional demands of the visual task (high or low) and the side of visual attention (same as or opposite to the relevant auditory sequence). On the basis of Driver and Spence's (1994) study, we expected to see a cost of increased attentional demands (i.e., performance on the auditory task being worse when it was concurrent with the more attentionally demanding visual task). The critical prediction, however, was that auditory-shadowing performance would be modulated by the relative direction of visual attention. That is, shadowing performance for auditory words would be worse when the participants had to shadow the words from one side while performing the visual detection task on the other side than when both relevant sources of information came from the same side.

Although similar to the task used by Driver and Spence (1994), there were some noticeable differences. First, the words in the shadowing task were presented here at a constant rate and not in triplets, in the expectation that this would induce a sustained attention state in which the participant would maintain the effort to continuously focus on the relevant auditory sequence (instead of attempting to reinitiate an attention shift prior to each triplet). Second, visual detection performance was monitored in both visual conditions. The less demanding visual task was a zero-back task (i.e., a simple detection task with a single target). The harder task was a one-back task in which a target consisted of any letter presented twice consecutively in the visual sequence. Thus, a gauge of visual attention was obtained in all the conditions, and the manipulation of visual attention could be assessed independently of its effects on auditory-shadowing performance. Third, the content of the visual displays was identical across the two levels of visual attention. This would ensure that any differences between passive and active tasks could not be attributed to potential differences in exogenously driven (i.e., involuntary) attention shifts. Finally, instead of stopping the shadowing task in order to respond verbally every time a visual target was detected, the participants responded to visual targets with a keypress while continuously shadowing the auditory message.

\section{Method}

Participants. Sixteen undergraduates (13 females and 3 males; mean age, 21.2 years, $S D=4.7$ years) with English as their native language participated in the experiment for course credit. All had normal or corrected-to-normal vision and normal hearing.

Apparatus and Materials. Two loudspeakers, one to the left and one to the right (at a distance of $57^{\circ}$ in azimuth between them), were used to present the auditory sequences. The loudspeakers were connected to the left and right channels of the sound card of a Pentium PC (Computer A). A third loudspeaker placed centrally emitted white noise at $56 \mathrm{~dB}$ (A) SPL, in order to make the shadowing task difficult enough. The visual stimuli were projected onto the surface of the loudspeakers, using an NEC MultiSynch LT100 projector connected to a second 486 PC (Computer B) controlling the sequence of visual stimuli. Computer A controlled the sequence of the experiment, played the auditory stimuli, registered the responses, and triggered the presentation of visual targets via a signal sent to Computer B through the serial port. The participant used a chinrest placed $64 \mathrm{~cm}$ from the center of the setup, with the head facing forward. A microphone was taped to the base of the chinrest and was connected to headphones through a Danelectro MiniAmp, in order to allow the experimenter to hear clearly the verbal responses and to track auditory-shadowing performance during the experiment. The experimenter monitored fixation and head position through a small mirror showing the upper half of the participant's face.

The auditory materials were the same words as those used by Driver and Spence (1994) but rerecorded by a female Canadian English speaker. Although the words were not presented in triplets, the original pairings used by Driver and Spence (1994; the 2 words that were presented simultaneously to the right and to the left speakers) were preserved, since they were matched on spoken frequency (according to Brown, 1984) and number of letters (see Driver \& Spence, 1994, for details). Some of the words from the original list were eliminated together with the other member of the pair, since they were words commonly used in England, but not in North America (e.g., lorry). We constructed eight experimental lists, each consisting of 36 word pairs, so that a total of 576 words were used. The individual words were recorded digitally as they were read aloud by a female speaker and were presented at an intensity of approximately $60 \mathrm{~dB}$ (A) as measured from the participant's head. The set of visual stimuli consisted of the uppercase letters B, C, D, F, G, H, K, L, M, N, $\mathrm{P}, \mathrm{Q}, \mathrm{R}, \mathrm{S} \mathrm{T}, \mathrm{V}, \mathrm{W}$, and X, appearing in white on a dark background and subtending $3.3^{\circ} \times 1.6^{\circ}$ of visual angle.

Procedure and Design. The experimental room was sound attenuated and remained dimly lit during the experiment. The participant sat at a table, with the head placed in the chinrest and the index finger of the dominant hand resting on the space bar of a keyboard. In every block, the 36 pairs of words were presented (one to the right and one to the left) in sequence at a constant rate of 1 pair every $1,000 \mathrm{msec}$. Prior to presentation of each experimental list, the participant was informed as to which loudspeaker to shadow from and onto which loudspeaker the visual display would be projected. He or she was also told about the nature of the visual task (zero-back or one-back). The visual stimuli (letters) were presented in a rapid serial visual presentation (RSVP) sequence (56-msec duration per item and 40-msec interstimulus interval). In the zeroback task, the participant monitored the RSVP sequence for the letter V. In the one-back task, the letter V never appeared, and the participant monitored the RSVP sequence for any letter repeating back to back. Visual targets appeared three times during each block; each target was inserted at a random serial position into the first, second, or third part of the list. 
Two additional lists of 16 word pairs were used for practice and were administered to the participant prior to the start of the experiment. The eight experimental lists were then administered twice, with the relevant and the irrelevant channels swapped in the second run, so that the participant shadowed each of the words once. The order of the eight conditions (resulting from crossing the factors side of shadowing, the side of visual attention, and the visual task) was arranged according to a Latin square that was repeated twice in reverse order for every participant. Two participants were tested with each of the resulting eight different orders.

\section{Results}

Separate analyses of variance (ANOVAs) were conducted on auditory (shadowing) and visual (target detection) performance.

Auditory shadowing. Auditory-shadowing performance was computed as the mean percentage of words correctly repeated for each participant in each condition (see Figure 1). We accepted close morphological derivations as correct answers (e.g., cats for cat) but considered other types of approximation as incorrect answers (e.g., hat for cat). A two-way within-subjects ANOVA of shadowing performance for auditory words was conducted that included visual attention (high in the oneback task vs. low in the zero-back task) and side (same vs. opposite with respect to the side of fixation as factors). The analysis indicated a main effect of visual attention, with auditory-shadowing performance at $42 \%$ for the zero-back task and at $36 \%$ for the one-back task $[F(1,15)=19.55, p<.0005]$. There was no effect of side on auditory-shadowing performance $(M=40 \%$ for the same-side condition, and $M=39 \%$ for the oppositeside condition; $F<1$ ). Finally, the interaction between visual attention and side did not reach significance $(F<1)$. An additional analysis including the side of the display (right vs. left) was conducted, but it failed to reach significance, and display side did not interact with any other factor (all $F \mathrm{~s}<1$ ). Therefore, we collapsed the data across display side, and as in Driver and Spence (1994), will report only the two factors of interest.

Visual detection. The analysis of visual detection included the two within-subjects factors: side (the visual display could be on the same side as the relevant auditory source or on the opposite side) and visual attention (low attention demands in the zero-back task or high attention demands in one-back task). There was a significant effect of side $[F(1,15)=5.0, p<.05]$, with same-side targets being detected more frequently than opposite-side targets $(M=56 \%$ vs. $M=49 \%)$. There was also a marginal effect of visual attention $[F(1,15)=4.0, p<.07]$ : Visual targets were detected with $59 \%$ accuracy in the zero-back task and with $46 \%$ accuracy in the one-back task. Additional analyses including side of visual display (left or right) failed to show additional main effects or interactions (all $F_{\mathrm{S}}<1$ ), and in subsequent experiments, the data were collapsed across display side.

\section{Discussion}

As was expected, auditory-shadowing performance suffered a decrement as a function of the attentional de- mands of the secondary visual task. However, contrary to our predictions, no evidence for costs related to attending to spatially separate auditory and visual sequences was found in the shadowing task. This is clearly different from what Driver and Spence (1994) found (see also Spence, Ranson, \& Driver, 2000; Spence \& Read, 2003), since it suggests that people can move their auditory attention away from where they are attending visually, apparently without cost to performance. In the visual task, there was an effect of attentional demands (performance in the zero-back task was better than that in the one-back task), although this effect (13\%) was only marginally significant. We also found an overall cost in visual detection performance when auditory and visual attention were divided across different locations.

One surprising result in this experiment was the failure to obtain a modulation of auditory-shadowing performance as a function of the spatial allocation of visual attention. This result is interesting in the context of the existing literature suggesting links between vision and audition in endogenous spatial orienting. There are reasons to believe that this attention link exists (Driver \& Spence, 1994), but something in the present experiment has apparently caused it to disappear, perhaps by masking it or by shifting the burden of processing to the visual modality in order to compensate for the reduced amount of resources available to process the auditory information. Indeed, one tempting explanation for this result is that the cost of dividing attention to different locations across modalities was there but that, in the present case, the participants sacrificed visual detection accuracy (where a spatial cost was observed) in order to maintain the accuracy level in the auditory-shadowing task. However, this must remain a highly speculative account at the moment, because in terms of the experimental design, the visual task was secondary (i.e., it contained only a limited number of targets used to ensure and monitor that the participants were effectively attending to the visual stream). In any case, note that the fact that there was no interaction between the visual spatial cost and attention load would render an explanation based on this speculation incomplete.

As was discussed earlier, we introduced a series of methodological changes with respect to Driver and Spence's (1994) work. Therefore, we began by examining the differences between the present task demands and those present in the paradigm used by Driver and Spence (1994), where a cost of directing visual and auditory attention to opposite locations was observed on auditoryshadowing performance. The lack of evidence for a crossmodal attention effect on shadowing in Experiment 1 could result from differences in the attentional demands required by the visual task and/or the auditory task, as compared with the tasks in Driver and Spence (1994). Indeed, in one of their experiments, Spence, Ranson, and Driver (2000) failed to find the expected increase in interference from auditory distractors at the location of visual attention when task demands were not sufficiently high. Here, in Experiment 1, performance levels in the two tasks were within measurable range (i.e., there were 


\section{A - Auditory-shadowing performance}

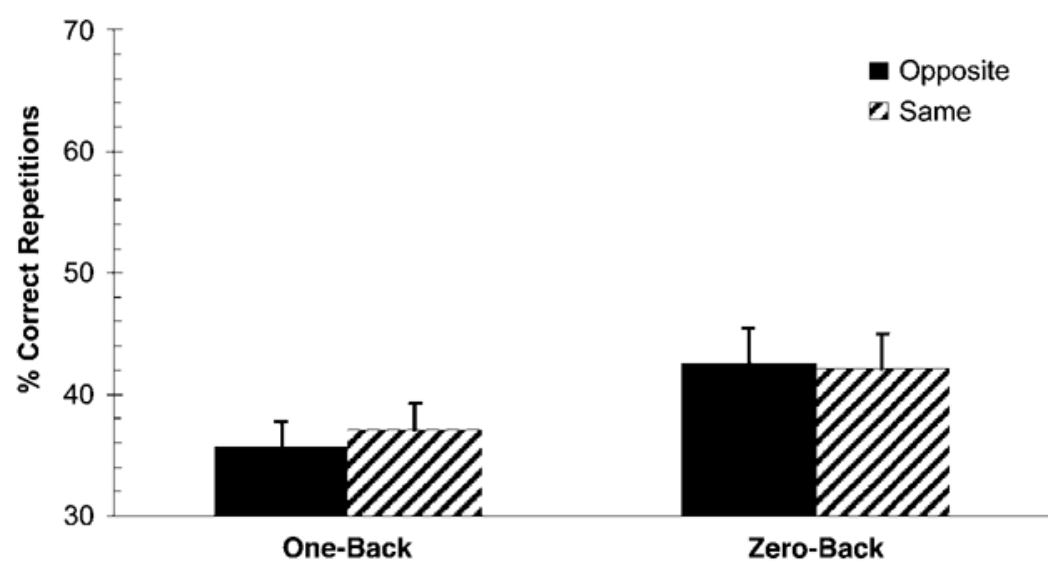

\section{B - Visual detection performance}

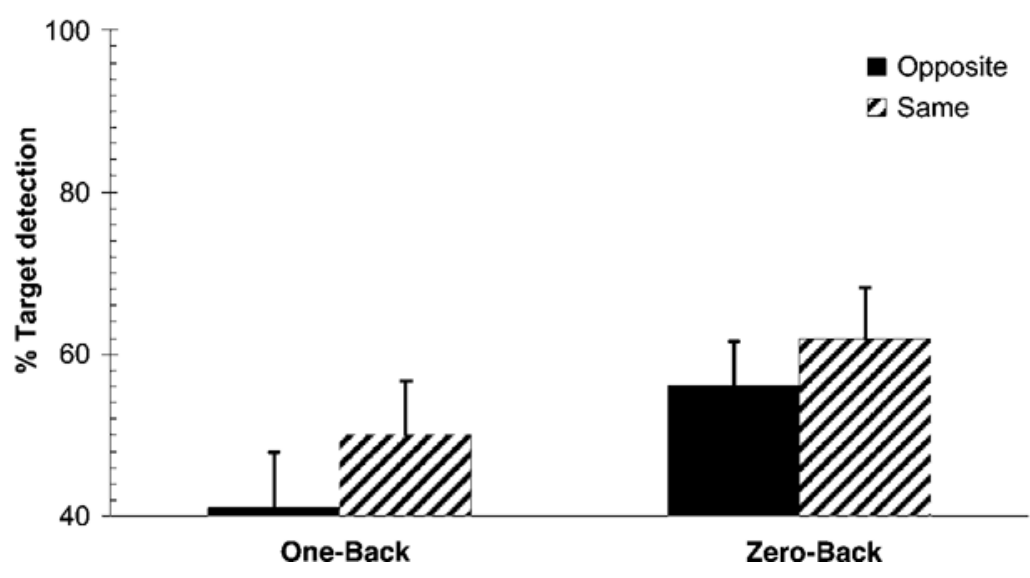

Figure 1. Average performance on the shadowing task (panel $A$ ) and on the visual task (panel B) in Experiment 1. Results are presented as a function of visual task (oneback vs. zero-back). Hatched bars represent performance when the participants were shadowing from the location of visual attention, and solid bars represent performance when they were shadowing from the location opposite to the direction of visual attention. Line bars represent the standard errors of the means.

no possible floor or ceiling effects), but they were somewhat different from those obtained by Driver and Spence (1994). Performance on the visual task $(M=53 \%)$ was lower overall than that reported by Driver and Spence (1994, Experiment 2; $M=79 \%$ ), although it was at the same level as that in Spence, Ranson, and Driver (2000, Experiment 3; $M=52 \%$ ), where the expected cost on auditory-shadowing performance was observed. Moreover, with regard to performance on the shadowing task, accuracy in the present Experiment $1(M=39 \%)$ was somewhat lower overall than that in Driver and Spence (1994; $M=48 \%$ ) and slightly lower than that in Spence, Ranson, and Driver (2000, Experiment 3; $M=44 \%$ ). In summary, performance in the visual task and in the shadowing task was equivalent or slightly worse in our Experiment 1 than in the cited studies, therefore ensuring that the tasks used here were demanding enough (although note that they were still very far from floor performance). It is unclear, therefore, how the increased difficulty in the visual and the auditory tasks would reduce, rather than enhance, audiovisual links in spatial attention. ${ }^{2}$

Another potentially important factor is that our lowattention condition still required some attentional resources, since the participants needed to constantly keep track of the visual displays in search for the target, whereas in Driver and Spence's (1994) study, it consisted of passively looking at the visual display. This fact may account for the finding of no interaction between the low- and the high-attention conditions, provided that an overall cost of dividing attention across spatial locations was found. However, it is difficult to see how the absence of a truly passive condition may have produced the lack of spatial costs in auditory performance, the result found here. Finally, it is worth noting that we found a clear overall nonspatial modulation related to increasing visual demands on auditory-shadowing performance. The size of this overall divided attention cost on auditory-shadowing performance in Experiment 1 (8\% cost) was identical, in 
numerical terms, to that obtained by Driver and Spence $(1994 ; 8 \%)$. Nevertheless, in the next experiment, we used the same passive and active visual tasks as those in Driver and Spence (1994; although this meant losing the measure of visual detection performance for the passive task).

\section{EXPERIMENT 2}

Since the previous experiment was unsuccessful in replicating the spatial synergy effect between visual and auditory attention (at least in terms of costs in auditory shadowing, our primary measure), we sought to change the visual task in order to make it more similar to the one used in Driver and Spence (1994). Although the overall percentage of visual accuracy was somewhat lower than that in Driver and Spence (1994; suggesting that the visual task was demanding enough), we speculated that one potentially important factor for our failure to replicate would be that, in our experiment, even the low-attention condition required visual attention. Although the high- and the low-attention conditions certainly required different degrees of attention, the fact that we did not include a passive task may have made any noticeable modulation disappear on the relatively crude measure of shadowing.

We used visual tasks similar to those used by Driver and Spence (1994), constituting a passive condition, in which participants merely fixated the RSVP sequence, and an active condition, in which participants searched for a + sign amid an RSVP of different keyboard symbols. The auditory task remained unchanged. Thus, in this experiment, the differences in the visual task were targeted as, perhaps, leading to the breakdown or masking of the possible synergy between endogenous auditory and visual attention. In addition, in their experiment, Driver and Spence (1994) measured the proportion of intrusions of stimuli presented at the unattended auditory sequence (i.e., the number of times that the participants erroneously shadowed the word presented at the irrelevant location). Upon reexamining the performance of the participants in Experiment 1, it was noted that intrusions were uncommon, yet no systematic measure had been obtained. In the present experiment, intrusions were also measured, since it was possible that an effect would emerge with more intrusions when the visual and the shadowing tasks were on opposite sides, reflecting auditory attention being unavoidably directed toward the location of the visual task. Therefore, the dependent measures included shadowing accuracy, shadowing intrusions, and visual accuracy in the active task.

\footnotetext{
Method

Participants. Sixteen new undergraduates (12 females and 4 males; mean age, 21.1 years, $S D=3.4$ years) with English as their native language participated in the experiment for course credit. All had normal or corrected-to-normal vision and normal hearing.

Apparatus, Materials, and Procedure. The apparatus and the materials were identical to those in Experiment 1, with the following exceptions. The speakers from which the words were presented were of a different make (Koss Corporation, Milwaukee, WI). The visual stimuli were changed so as to be in accordance with those
}

used by Driver and Spence (1994). In this experiment, the visual RSVP sequence was composed of a random sequence of the keyboard symbols $<,>, !,(), *,,\{\},,[],,-, /,:, \&$, and $\mid$.

The procedure and design were the same as those in Experiment 1, except that instead of the zero-back and one-back tasks, the visual tasks were now passive and active tasks, respectively. In the active task, the participants were asked to detect the + symbol, which was inserted in the sequence pseudorandomly (with the same restrictions as those described in Experiment 1). In the passive task, the participants were asked simply to look at the sequence, which never contained the + symbol.

\section{Results}

Auditory-shadowing performance. Individual accuracy was measured for every condition (see Figure 2), and an ANOVA was conducted with the within-subjects factors of side (same or opposite side as visual fixation) and visual attention (passive vs. active). The results indicated a main effect for visual attention $[F(1,15)=40.8, p<$ $.001]$, with auditory-shadowing performance at $51 \%$ for passive fixation and at $45 \%$ for the active task. There was no effect for direction of fixation relative to the shadowing side $(F<1)$, with mean auditory-shadowing performance of $48 \%$ when the participants fixated at the location of relevant sounds and $48 \%$ when the participants fixated at the opposite location (i.e., at the location of the irrelevant sounds). The interaction also did not reach significance $(F<1)$.

Intrusions. We assessed the percentage of words from the irrelevant auditory list that were erroneously shadowed by the participants. However, they proved to be quite rare and averaged less than $2 \%$ overall, and a meaningful analysis of the intrusions could not be conducted.

Visual detection performance. Detection performance could be examined only for the active task, since no measure was obtained in the passive task. No effect was found for the side of visual attention as a function of the side of auditory attention $(F<1)$. Visual detection was at $83 \%$ when the visual task was on the same side as the relevant word sequence and at $82 \%$ when it was on the opposite side.

\section{Discussion}

No evidence for cross-modal links in spatially attending separate auditory and visual sequences was found in any of the three measures used (shadowing accuracy, intrusions, and visual detection accuracy). Although unsuccessful at replicating reports of spatial synergy, the present performance levels in both the visual and the auditory tasks were very similar to those in Driver and Spence (1994). Note, for instance, that overall performance here was $48 \%$ in the shadowing task and $83 \%$ in the visual task, whereas Driver and Spence (1994) obtained 48\% and $79 \%$, respectively. In addition, the overall influence of the visual task on auditory-shadowing performance (6\% effect) was significant and very similar to the one observed by Driver and Spence (1994; 8\% effect). However, there are still a number of differences that could be important in explaining why the present manipulation failed to produce any synergies even if the overall performance and stimu- 


\section{A - Auditory-shadowing performance}

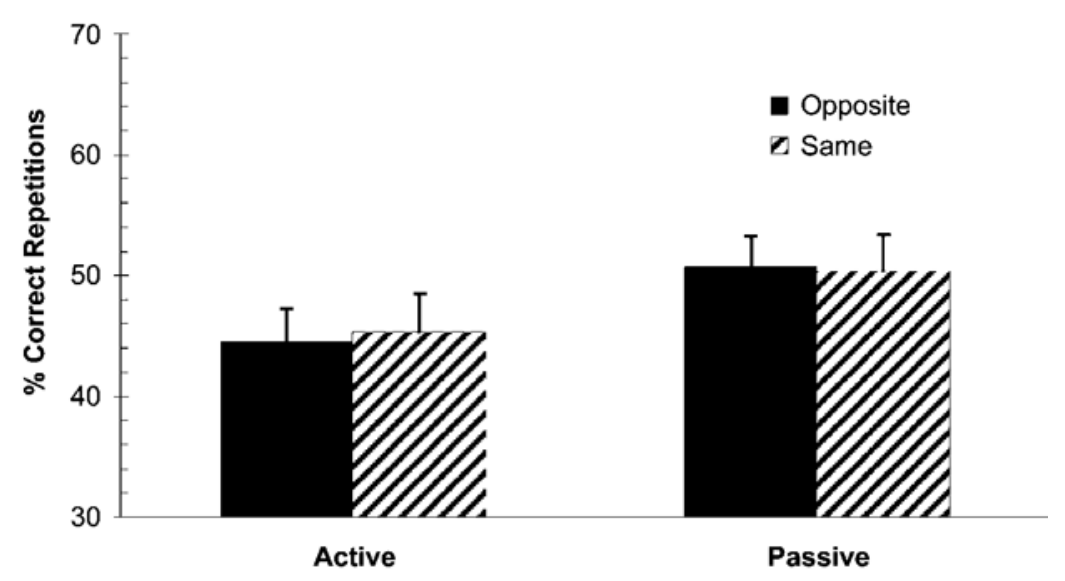

\section{B - Visual detection performance}

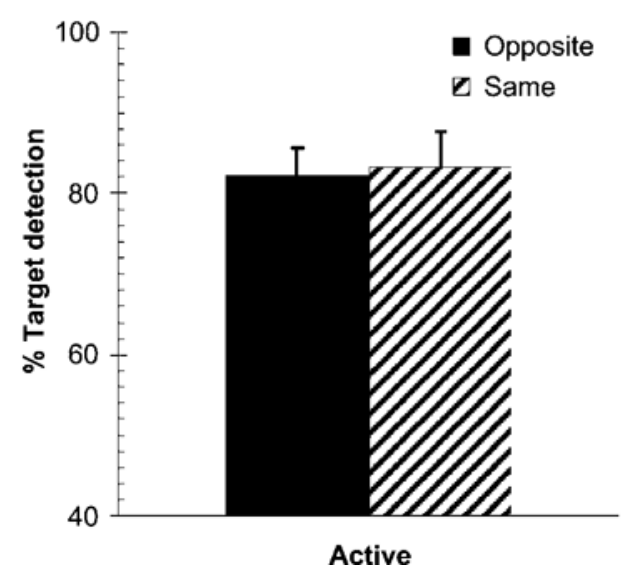

Figure 2. Average performance on the shadowing task (panel A) and the visual task in the active condition (panel B) in Experiment 2. Results are presented as a function of visual task (active vs. passive). Hatched bars represent performance when the participants were shadowing from the location of visual attention, and solid bars represent performance when they were shadowing from the location opposite to the direction of visual attention. Line bars represent the standard errors of the means.

lus conditions were closely matched to those used by Driver and Spence (1994).

Perhaps, one important difference between the present study and Driver and Spence (1994), as well as subsequent studies adapting their paradigm, is that the visual displays they used for the active and the passive conditions were physically distinct. This difference is potentially very relevant, given that the displays used in previous studies for the active condition have usually contained more stimulus onsets/offsets and more variability than the displays used in the passive task did. This could explain the extra attentional engagement in the active conditions (and in consequence, the extra attention to the location of the relevant or irrelevant sounds, depending on the condition) that would be necessary to observe the synergies. In our Experiments 1 and 2, the displays used in the passive and the active conditions (or high vs. low attention) were physically identical, and in principle, there could not be a difference in the degree to which exogenous attention was engaged across conditions.

Another potentially important factor, already discussed in the introduction, is that Driver and Spence (1994), as well as subsequent studies, presented the auditory materials in triplets, with the participants responding during a silent period after the triplet of words had been presented. This may have induced the participants to reinitiate a divided attention state anew prior to each triplet. If this were the case, Driver and Spence's (1994) paradigm may have underestimated the participants' true ability to split attention between modalities and locations in a sustained attention state. In our experiments, shadowing conformed to the traditional use of the term, whereby the participants repeated the words as they heard them, without pauses in the input stream. Accordingly, we assume that in Experiments 1 and 2, our participants were less likely to initiate multiple attention 
shifts and sustained their attention over the whole block. Finally, another possibly relevant difference between Driver and Spence's (1994) study and ours was the output mode in the visual task. Whereas Driver and Spence (1994) required verbal responses to the visual targets (thereby interrupting the shadowing task), only manual responses were required in our studies. ${ }^{3}$ In the next experiment, we attempted a close replication of Driver and Spence's (1994) Experiment 2.

\section{EXPERIMENT 3}

The main goal of this experiment was to replicate the second experiment in Driver and Spence (1994) as closely as possible. It may be that when auditory stimuli are presented in triplets with intervening pauses, auditory attention is released, and the resulting disengaging and reengaging of auditory attention produces a synergy effect (possibly by making auditory attention more vulnerable to other ongoing attentional processes). In addition, Experiments 1 and 2 required a manual keypress response to visual targets, rather than a verbal response (as used in Driver and Spence, 1994). This may be important because, as the two tasks become increasingly similar (in terms of output mode), more interference may occur, and therefore, the synergy could arise more easily when the responses to both tasks share the same effectors. Although there is no obvious reason for why this output interference should be stronger in the same-side conditions than in the opposite-side conditions, it is important to address the potential role of response modality in the establishment of cross-modal synergies (e.g., Wickens, 1980, 1984). Thus, in the present experiment, the participants were asked to pause shadowing and say "plus" out loud when detecting a visual target.

Several additional changes to the procedure were introduced in order to match it as closely as possible to the procedure used in Driver and Spence (1994). The number of visual targets per list was changed to two, three, or four, so as to be more similar to Driver and Spence's (1994) original study. Also, the stimuli in the passive task were changed to the letters $\mathrm{Z}$ and $\mathrm{O}$, whereas the stimuli for the active task remained the same as those in Experiment 2. The order of the tasks was constructed to replicate the original design, and the side from which to shadow was alternated on a block-by-block basis.

\section{Method}

Participants. Sixteen new undergraduates with English as their native language participated in the experiment for course credit: 12 females and 4 males, mean age 20.8 years $(S D=3.3$ years), all but 2 right-handed. All had normal or corrected-to-normal vision and normal hearing.

Apparatus, Materials, and Procedure. An RCA SA-155 amplifier was used, instead of the miniamplifier used in Experiments 1 and 2 . The passive visual task was changed so that now it consisted of the random presentation of the characters $\mathrm{O}$ and $\mathrm{Z}$ at the same rate as that of the RSVP sequence in Experiments 1 and 2. The side of visual attention was alternated from one list to the next, the rest of the factors being counterbalanced around this constraint, as in
Driver and Spence (1994). The words were now presented in triplets, with one word appearing approximately $540 \mathrm{msec}$ after the onset of the previous one within one triplet and with an interval of $4,000 \mathrm{msec}$ between the end of one triplet and the beginning of the next, to allow for the participants' responses. The number of triplets per list was 12 (instead of the 14 used by Driver \& Spence, 1994, because of the eliminated words; see Experiment 1, Method).

\section{Results}

Auditory-shadowing performance. Individual accuracy was measured for every condition (see Figure 3), and an ANOVA was conducted with side (same or opposite side as in the visual task) and visual task (passive vs. active) as factors. The ANOVA indicated a significant effect for visual task, with shadowing accuracy in the passive condition (54\%) being higher than that in the active condition $[41 \% ; F(1,15)=141.2, p<.001]$. The effect of the side of the visual task was not significant, and the mean was $48 \%$ for the same side and $47 \%$ for the opposite side $[F(1,15)=1.7, p=.298]$. The interaction between visual task and side did not reach significance $(F<1)$.

The analysis above took as its dependent measure an absolute measure of accuracy - that is, out of all the words correct, how many did the participant manage to shadow? However, since the visual task required the participant to stop shadowing for certain triplets, it is possible that a better measure of shadowing would be performance for triplets only when the subject did not stop shadowing and indicate that a target was present. An analysis of auditory-shadowing performance from triplets for which no visual response was required yielded a nearly identical pattern, with a significant difference in auditoryshadowing performance as a function of visual task [ $M=54 \%$ in the passive condition and $M=46 \%$ in the active condition; $F(1,15)=52.6, p<.001$ ], no effect of side $(M=51 \%$ for the same-side condition and $M=$ $49 \%$ for the different-side condition) and no interaction ( $F<1$ for both).

Intrusions. An ANOVA on intrusions (including the same factors) revealed a marginal effect for side $[F(1,15)=4.1, p=.07]$, with $2.7 \%$ intrusions for the same side and $3.2 \%$ for the opposite side. No other effects approached significance in this analysis.

Visual detection performance. Accuracy in the visual detection task was obtained only in the active task. Again, no difference was found between the same- and opposite-side conditions, with $78 \%$ for the same side and $79 \%$ for the opposite side $(F<1)$.

\section{Discussion}

The results of Experiment 3 confirmed the results of Experiment 2 in most ways. Thus, the presence of a continuous sequence of words versus the presentation of triplets was not the crucial factor for inducing spatial synergy. Neither did using the same response mode (i.e., verbal) for both the auditory and the visual tasks account for the lack of a synergy effect on shadowing performance. The difference in shadowing accuracy for the ac- 


\section{A - Auditory-shadowing performance}

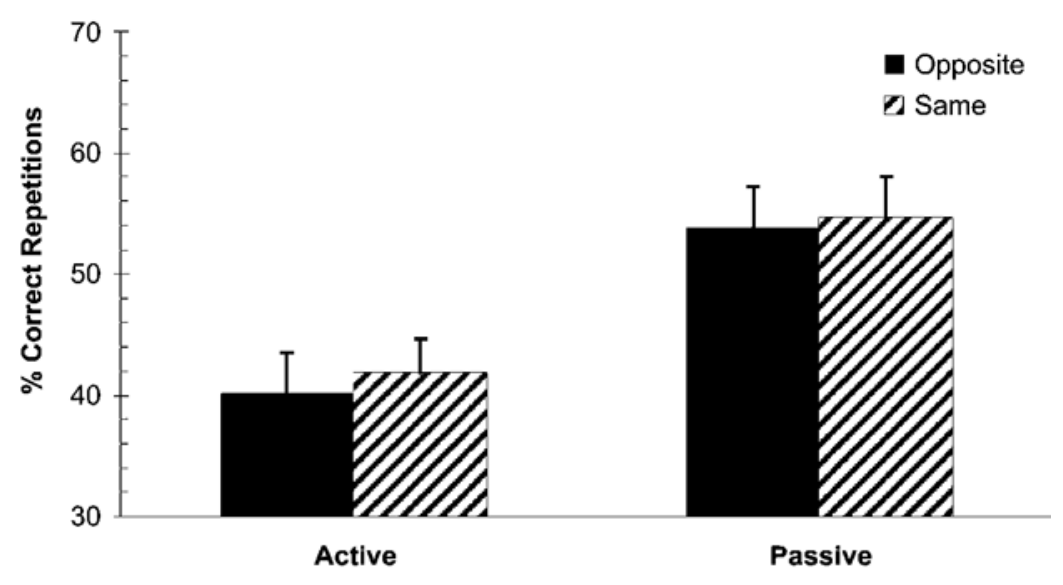

\section{B - Visual detection performance}

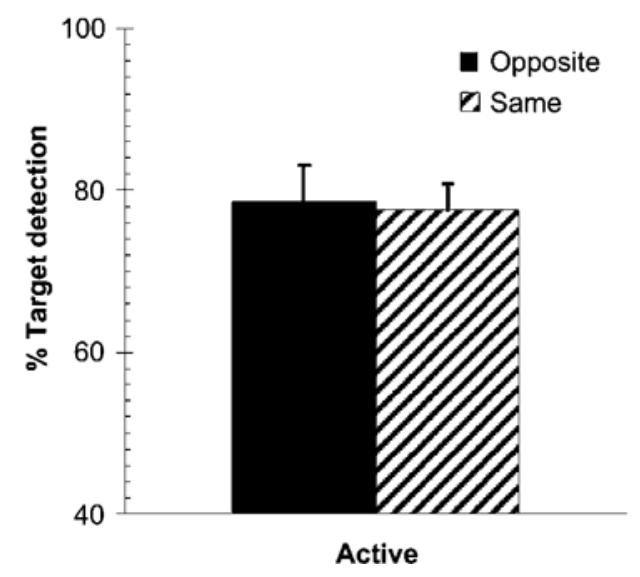

Figure 3. Average performance on the shadowing task (panel $A$ ) and the visual task in the active condition (panel B) in Experiment 3. Results are presented as a function of visual task (active vs. passive). Hatched bars represent performance when the participants were shadowing from the location of visual attention, and solid bars represent performance when they were shadowing from the location opposite to the direction of visual attention. Line bars represent the standard errors of the means.

tive versus the passive visual tasks was larger than that in the previous experiment. It is possible that the physical difference between the visual displays in the active and the passive conditions in this experiment accounts for the larger difference between the two tasks. Note that overall, the performance accuracy in the shadowing task $(M=$ $48 \%)$ and in the visual detection task $(M=78 \%)$ were, again, effectively identical to those obtained in Driver and Spence (1994, Experiment 2): $48 \%$ and 79\%, respectively.

For the first time, we found a slight tendency toward a difference between same and different sides on auditoryshadowing performance - in particular, on the percentage of intrusions. However, it is only fair to note that this marginally significant effect was very small $(0.5 \%)$, and its possible importance may be overestimated in the statistical tests because of the reduced variability of percentage measures when the scores are very close to the lower end of the distribution (see Howell, 1982), as was the case here (3\% intrusions, overall). ${ }^{4}$ But most important, this trend in the main factor of side was not a function of visual task (i.e., there was no trace of an interaction), and therefore, it cannot be taken as an indication of a purely attentional effect.

At this point, only very marginal differences remain between Driver and Spence's (1994) Experiment 2 and the present attempt to replicate it. For example, there were slightly fewer words per list (because we had to take out the non-North-American English words), the apparatus and population were obviously different, and the actual speaker whose voice was recorded was also different. However, if these are the kinds of differences that are to account for the failure to replicate, the effect is indeed so fragile as to negate any attempt to build reasonable suppositions on it. 


\section{GENERAL DISCUSSION}

The most robust finding to emerge from this study is that participants seem to be able to select a continuous auditory message and monitor a sequence of visual items at opposite locations with more ease than could have been predicted from earlier findings. This ability to divide auditory and visual attention is striking in light of the extensive cross-modal links demonstrated in past research, regarding both exogenous (involuntary) and endogenous (voluntary) spatial attention (see Driver \& Spence, 2004, and Spence \& McDonald, 2004, for recent reviews). In all of the experiments presented in this study, the tasks were sufficiently demanding and of comparable difficulty with those used in previous studies. Dual-task costs were clearly observed in auditory performance in the shadowing task, and these were more pronounced the more demanding the visual task. It is clear that the present data do not prove the absence of audiovisual links during sustained attention, but they clearly indicate that when given time, humans have a remarkable ability to split their auditory and visual attentional resources across different spatial locations even under difficult conditions.

\section{Shifting Versus Sustaining Attention}

The present results concern a situation that occurs often in real life - namely, observers had to maintain a divided attention state over a prolonged period of time, as opposed to shifting attention from trial to trial. This may be a potentially important issue for understanding the apparent decoupling of auditory and visual attention in our study and its discrepancy with other cross-modal studies (but see also Alfonso, unpublished data, cited in Pashler, 1997, p. 91; Duncan et al., 1997). In most of the past experiments demonstrating audiovisual links in spatial endogenous attention, the methodology induced participants to reorient to a central location before each new trial was initiated (for example, by means of a central fixation cue). As was discussed in the introduction, some authors have pointed out the distinction between phasic (transient) and tonic (sustained) attention as an important factor in the cross-modal attention literature (Eimer \& Van Velzen, 2002). It is interesting, for example, that several authors have found electrophysiological evidence for tactile attention effects as a function of visual or auditory attention shifts only in paradigms leading to phasic orienting, but not under sustained attention states (Eimer \& Driver, 2000; Eimer et al., 2002). These results suggest that people can arrive at optimal divided attention performance. However, it is perhaps premature to claim that cross-modal links in attention are mandatory during phasic attention shifts but may be largely overridden during sustained attention states, and this must remain as a conjecture at this point. Indeed, there is now some evidence against this view for the case of auditory-tactile attention (Hötting et al., 2002). Therefore, although the distinction between phasic and sustained attention states may be an important aspect in the establishment of cross- modal attention links, its specific role needs to be addressed in the future. ${ }^{5}$ In any case, the arguments regarding the distinction between phasic and sustained attention shifts are insufficient to explain why the results obtained in the present study differ from the results obtained by Driver and Spence (1994) — in particular, those in our Experiment 3 (and the additional experiment reported in the Appendix), where the auditory task was delivered in triplet format.

\section{A Weak Cross-Modal Link}

What could be the reasons for the present failure to replicate Driver and Spence's (1994) synergy? We have already ruled out some obvious candidates. For instance, as Spence, Ranson, and Driver (2000) highlighted, it could be that the synergy disappears when the visual task is not demanding enough (their Experiment 2). In our experiments, the level of visual detection performance was equivalent or even slightly lower than that observed in Driver and Spence (1994; see also Spence, Ranson, \& Driver, 2000, Experiment 3). A second potential reason could be that Driver and Spence (1994) used bright LED displays for their visual task, whereas we used an LCD projection (white items on a dark background screen) that had a high contrast, but perhaps not as much luminance as an LED display. However, if this is a key factor in explaining the difference in results, Driver and Spence's (1994) effect may have been in part due to exogenous (i.e., involuntary) or bottom-up attentional capture of the visual display, as opposed to the consequence of directing voluntary attention to one or the other side. This potential confound becomes a serious concern because, in all the applications of Driver and Spence's (1994) methodology to date (except our Experiments 1 and 2), the content of the visual displays has been different in the active and the passive conditions. A third potential reason for the discrepancy between the results in Driver and Spence (1994) and the present results may be a difference in instructions. As was the case in Driver and Spence's (1994) study, here the participants were not informed about the exact probability of occurrence of the visual targets but were told that they would occur only in active conditions (here, the latter is relevant only to Experiments 2 and 3). Nevertheless, although some differences in instructions are possible, it is also the case that overall performance in the two tasks mirrored that reported in Driver and Spence (1994). Moreover, as was noted above, such differences would point merely to the fragility of the phenomenon.

Another possible factor worth examining is practice. There is a good deal of evidence for the dramatic effects that extensive practice can have on attention (e.g., Braun, 1998), and one of the typical benefits of practice is to eliminate or reduce interference from distractors and to improve selective attention. That said, it is unlikely that practice could explain the differences between Driver and Spence's (1994) results and those in the present study. First, our participants had fewer, and not more, practice trials (this was for purely practical reasons, since we had fewer stimulus words). We nevertheless ex- 
amined whether an effect of practice was present in the initial part of our experiments and conducted an analysis including the factor of block (first vs. second part of the experiment) for each of our three experiments. Although there was an overall practice effect, with the second half of the experiment yielding better shadowing than the first half $\operatorname{did}[37 \%$ vs. $42 \%$ in Experiment $1, F(1,15)=10.1$, $p<.01 ; 45 \%$ vs. $51 \%$ in Experiment $2, F(1,15)=45$, $p<.001$; and $45 \%$ vs. $50 \%$ in Experiment $3, F(1,15)=$ $23.07, p<.001]$, in none of the experiments did this factor interact with side $(F<1$ for Experiments 1 and 2, and $F=1.7, p>.2$ in Experiment 3$)$.

Localizability has also been demonstrated as a potentially important factor for the replication of another crossmodal attention result (Driver, 1996). Leech (1999) found that the precise degree of localizability of the auditory stimuli (manipulated on the basis of spatial separation) was critical for the replication of the findings in Driver (1996). However, localizability does not appear to account for the discrepancy between the results in Driver and Spence (1994) and the present results. Inspecting the proportion of intrusions as an overall indicator of localizability of the auditory information, our Experiment 3 produced an amount of intrusions similar to that reported in Driver and Spence (1994, Experiment 2).

Finally, the question arises as to the statistical power of our study to detect an effect like the one observed by Driver and Spence (1994), which was certainly small. The magnitude of Driver and Spence's effect in Experiment 2 was no bigger than $7 \%$. It could, therefore, be that our experiments lacked the power to tease out such a small effect. However, we believe that this is unlikely. In none of the three experiments reported (including the experiment in the Appendix) was there a trend in the expected direction in auditory-shadowing performance. Nevertheless, in an attempt to increase power, an ANOVA was performed on auditory-shadowing accuracy, pooling the data from all three experiments. The analysis showed that neither the effect of side $(45 \%$ in the same-side condition and $45 \%$ in the opposite-side condition; $F<1$ ) nor the interaction between side and visual task approached significance $(F<1)$. Note that the power in this analysis, assuming an expected effect size of $6 \%$ on the basis of Driver and Spence's (1994) Experiment 2, was $p>.90$, with $\alpha=0.05$.

Having highlighted the factors that could reasonably explain the present discrepancy, we are at a loss to explain why the small synergy effect was absent in our investigations, beyond the natural variability that most psychological measures have across different observers, laboratories, and equipments. ${ }^{6}$ This speaks to the weakness of the synergy effect during sustained attention and the possibility that people may be able to override it in order to perform optimally under certain circumstances. It is worth noting that auditory-shadowing performance is a relatively crude measure for such a fragile effect, and perhaps other behavioral or neuroimaging methodologies would be more appropriate and able to show that there are, indeed, limitations to dividing attention across modalities and locations. At this point, however, such limitations seem far from proven.

In an attempt to capture the subjective impressions of the participants about the different conditions in the experiments, we informally questioned them about which condition they felt was easier, after the experimental session. Almost always, the participants said that it was harder to shadow during the active (or one-back) task than during the passive (or zero-back) one. However, people did not consistently feel that it was easier to shadow on the same side as the visual task. In fact, several people said that it was easier to shadow the auditory sequence that was spatially separated from the visual one. These subjective reports seem to match the objective data analysis - namely, a clear dual-task cost, but no detrimental effect of spatially splitting visual and auditory attention.

\section{Implications for Current Accounts of Cross-Modal Attention}

There are at least two implications for our results. First, it seems that cross-modal links in spatial attention can sometimes be overridden (or, at least, greatly attenuated) when an attention state has to be sustained for a prolonged period of time. It is important to note that the only consistent indication of a cross-modal attention effect in the study revealed itself as a small effect of side of presentation in visual performance (Experiment 1), and even then, there was no indication that the degree of attention (low or high attention load) had any role. As is the case with cross-modal links in exogenous attention, cross-modal effects in endogenous attention do not seem to conform to a simple picture. For instance, several studies are suggestive of the idea that the more one paradigm approaches a truly sustained attention state, the smaller the chances of finding a synergy effect between certain combinations of modalities (see Eimer \& Driver, 2000, and Eimer et al., 2002, as well as the present study). However, this is not always the case (Hötting et al., 2002). In any event, regardless of the discrepancies between results, these studies seem to stress the importance of the separation between the consequences of phasic shifts of attention and tonic attention states as a potentially relevant factor when accounting for cross-modal attention results. It will certainly be interesting to see how these different results can be reconciled under one account, in the same way that some of the controversies regarding exogenous cross-modal attention appear to have finally been resolved (Spence \& McDonald, 2004; Spence, McDonald, \& Driver, 2004).

The second implication concerns the controversy about the mechanisms by which attention in different modalities is coordinated. Several authors have highlighted the possibility that, in spatial attention, there is a supramodal attention system by which all modalities are in register and access the same resources (e.g., Butter, Butchel, \& Santucci, 1989; Farah, Wong, Monheit, \& Morrow, 1989). On the other hand, some investigators have proposed that attention systems are separate for each modality but linked 
(e.g., Driver \& Spence, 1998; Spence \& Driver, 1996, 1997). A strict supramodal view of spatial attention would predict, among other things, that it is simply not possible to direct attention to different modalities at opposite locations without a considerable cost. In contrast with this prediction, our results suggest that it is possible in some circumstances, and they therefore support an account of cross-modal spatial attention with separate attention systems. These separate attention systems would, most of the time, work in a coordinated fashion, but not necessarily all the time. It is also important to note that the present results do not negate other, less strict versions of a supramodal account of attention.

\section{Conclusion}

We have shown here that endogenous spatial attention across modalities has a considerable degree of flexibility. Despite previous demonstrations about potential synergies in endogenous spatial attention between the auditory and the visual modalities, the link seems to be a fragile one. It must be noted that this conclusion is restricted to the case of sustained attention and may not apply to every case of sustained attention. But it is important to point out that at least under certain circumstances, humans are able to direct their visual and auditory attention to opposite spatial locations at no cost. In the future, it will be important to find the relevant factors leading to effective divided attention. In this respect, one potentially important aspect could be the similarity between the auditory and the visual stimuli. For example, note that in their Experiment 1, Driver and Spence (1994) used verbal material together with corresponding lip movements and found stronger synergies. However, as Driver and Spence (1994) noted (see also Spence, Ranson, \& Driver, 2000), those results were susceptible to confounds related to linguistic processing (see note 1). Most certainly, better knowledge of the situations leading to cost-free divided attention across modalities will be an important outcome in the design of human-operated devices and machines.

\section{REFERENCES}

Blauert, J. (1997). Spatial hearing (Rev. ed.). Cambridge, MA: MIT Press.

Braun, J. (1998). Vision and attention: The role of training. Nature, 393, 424-425.

Brown, G. D. A. (1984). A frequency count of 190,000 words in the London-Lund Corpus of English Conversation. Behavior Research Methods, Instruments, \& Computers, 16, 502-532.

Buchtel, H. A., \& Butter, C. M. (1988). Spatial attention shifts: Implications for the role of polysensory mechanisms. Neuropsychologia, 26, 499-509.

Butter, C. M., Buchtel, H. A., \& Santucci, R. (1989). Spatial attentional shifts: Further evidence for the role of polysensory mechanisms using visual and tactile stimuli. Neuropsychologia, 27, 1231-1240.

DRIVER, J. (1996). Enhancement of selective listening by illusory mislocation of speech sounds due to lip-reading. Nature, 381, 66-68.

Driver, J., \& SpenCE, C. J. (1994). Spatial synergies between auditory and visual attention. In C. Umiltà \& M. Moscovitch (Eds.), Attention and performance $X V$ : Conscious and nonconscious information processing (pp. 311-331). Cambridge, MA: MIT Press, Bradford Books.

Driver, J., \& SPEnce, C. (1998). Attention and the crossmodal construction of space. Trends in Cognitive Sciences, 2, 254-262.
Driver, J., \& Spence, C. (2004). Crossmodal spatial attention: Evidence from human performance. In J. Driver \& C. Spence (Eds.), Crossmodal space and crossmodal attention (pp. 179-220). Oxford: Oxford University Press.

Duncan, J., Martens, S., \& Ward, R. (1997). Restricted attentional capacity within but not between sensory modalities. Nature, 387, 808-810.

EIMER, M. (1996). ERP modulations indicate the selective processing of visual stimuli as a result of transient and sustained spatial attention. Psychophysiology, 33, 13-21.

Eimer, M. (1999). Can attention be directed to opposite directions in different modalities? An ERP study. Clinical Neurophysiology, 110, 1252-1259.

Eimer, M., \& Driver, J. (2000). An event-related brain potential study of cross-modal links in spatial attention between vision and touch. Psychophysiology, 37, 697-705.

Eimer, M., \& VAN Velzen, J. (2002). Cross modal links in spatial attention are mediated by supramodal control processes: Evidence from event-related potentials. Psychophysiology, 39, 437-449.

Eimer, M., VAN Velzen, J., \& Driver, J. (2002) Cross-modal interactions between audition, touch, and vision in endogenous spatial attention: ERP evidence on preparatory states and sensory modulations. Journal of Cognitive Neuroscience, 14, 254-271.

Farah, M. J., Wong, A. B., Monheit, M. A., \& Morrow, L. A. (1989). Parietal lobe mechanisms of spatial attention: Modality-specific or supramodal? Neuropsychologia, 27, 461-470.

GoPHER, D. (1973). Eye-movement patterns in selective listening tasks of focused attention. Perception \& Psychophysics, 14, 259-264.

Grant, K. W., \& Seitz, P. F. (1998). Measures of auditory-visual integration in nonsense syllables and sentences. Journal of the Acoustical Society of America, 104, 2438-2450.

Grant, K. W., \& Seitz, P. F. (2000). The use of visible speech cues for improving auditory detection of spoken sentences. Journal of the Acoustical Society of America, 108, 1197-1208.

Hillyard, S. A., Simpson, G. V., Woods, D. L., Van Voorhis, S., \& MÜNTE, T. F. (1984). Event-related brain potentials and selective attention to different modalities. In F. Reinoso-Suárez \& C. AimoneMarsan (Eds.), Cortical integration (pp. 395-414). New York: Raven.

Hötting, K., Rösler, F., \& RöDER, B. (2002). Crossmodal and intermodal attention modulates event-related brain potentials to tactile and auditory stimuli. Experimental Brain Research, 148, 26-37.

Howell, D. C. (1982). Statistical methods for psychology (3rd. ed.). Belmont, CA: Duxbury.

LABERge, D. (1995). Attentional processing. Cambridge, MA: Harvard University Press.

LEECH, S. (1999, October). Can the ventriloquism illusion facilitate audiovisual speech perception? Poster presented at the 1st annual meeting of the International Multisensory Research Forum (crossmodal attention and multisensory integration), Oxford.

Lloyd, D. M., Merat, N., McGlone, F., \& Spence, C. (2003). Crossmodal links between audition and touch in covert endogenous spatial attention. Perception \& Psychophysics, 65, 901-924.

PASHler, H. E. (1997). The psychology of attention. Cambridge, MA: MIT Press.

PosNer, M. I. (1980). Orienting of attention. Quarterly Journal of Experimental Psychology, 32, 3-25.

REISBERG, D. (1978). Looking where you listen: Visual cues and auditory attention. Acta Psychologica, 42, 331-341.

Reisberg, D., Scheiber, R., \& Potemken, L. (1981). Eye position and the control of auditory attention. Journal of Experimental Psychology: Human Perception \& Performance, 7, 318-323.

Soto-Faraco, S., Spence, C., Fairbank, K., Kingstone, A., Hillstrom, A. P., \& SHAPIRO, K. (2002). A crossmodal attentional blink between vision and touch. Psychonomic Bulletin \& Review, 9, 731-738.

Spence, C., \& Driver, J. (1996). Audiovisual links in endogenous covert spatial attention. Journal of Experimental Psychology: Human Perception \& Performance, 22, 1005-1030.

Spence, C., \& Driver, J. (1997). Audiovisual links in exogenous covert spatial orienting. Perception \& Psychophysics, 59, 1-22.

Spence, C., \& McDonald, J. (2004). The crossmodal consequences of the exogenous spatial orienting of attention. In G. Calvert, C. Spence, \& B. E. Stein (Eds.), The handbook of multisensory processes (pp. 325). Cambridge, MA: MIT Press. 
Spence, C., McDonald, J., \& Driver, J. (2004). Exogenous spatial cuing studies of human crossmodal attention and multisensory integration. In J. Driver and C. Spence (Eds.), Crossmodal space and crossmodal attention (pp. 277-320). Oxford: Oxford University Press.

Spence, C., Pavani, F., \& Driver, J. (2000). Crossmodal links between vision and touch in covert endogenous spatial attention. Journal of Experimental Psychology: Human Perception \& Performance, 26, 1298-1319.

Spence, C., Ranson, J., \& Driver, J. (2000). Crossmodal selective attention: On the difficulty of ignoring sounds at the locus of visual attention. Perception \& Psychophysics, 62, 410-424.

Spence, C., \& ReAD, L. (2003). Talking while driving: On the difficulty of splitting attention between eye and ear. Psychological Science, 14, 251-256.

SPERLING, G. (1960). The information available in brief visual presentations. Psychological Monographs, 74(11, Whole No. 498).

Sturm, W., de Simone, A., Krause, B. J., Specht, K., Hesselmann, V., Radermacher, I., Herzog, H., Tellmann, L., MullerGartner, H. W., \& Willmes, K. (1999). Functional anatomy of intrinsic alertness: Evidence for a fronto-parietal-thalamic-brainstem network in the right hemisphere. Neuropsychologia, 37, 797-805.

Sumby, W. H., \& Pollack, I. (1954). Visual contribution to speech intelligibility in noise. Journal of the Acoustical Society of America, 26, 212-215

Teder-Sälejärvi, W. A., MüNte, T. F., Sperlich, F., \& Hillyard, S. A. (1999). Intra-modal and cross-modal spatial attention to auditory and visual stimuli: An event-related brain potential study. Cognitive Brain Research, 8, 327-343.

Ward, L. M., McDonald, J. J., \& Lin, D. (2000). On asymmetries in cross-modal spatial attention orienting. Perception \& Psychophysics, 62, 1258-1264.

WicKeNs, C. D. (1980). The structure of attentional resources. In R. S. Nickerson (Ed.), Attention and performance VIII (pp. 239-257). Hillsdale, NJ: Erlbaum.

Wickens, C. D. (1984). Processing resources in attention. In R. Parasuraman \& D. R. Davies (Eds.), Varieties of attention (pp. 63-102). San Diego: Academic Press.

Wolters, N. C. W., \& Schiano, D. J. (1989). On listening where we look: The fragility of a phenomenon. Perception \& Psychophysics, 45, 184-186.

\section{NOTES}

1. It should be noted that Driver and Spence (1994) obtained stronger synergy results when using lip-read information in the visual display (a videotape of a person talking). However, as was argued by the authors themselves (see also Spence, Ranson, \& Driver, 2000), this situation is clearly subject to a potential confound with linguistic-specific processes, and hence, its generalization is limited. Therefore, we decided to focus on previous demonstrations using unrelated visual and auditory materials (words and keyboard symbols), just as in Driver and Spence
(1994, Experiments 2 and 3), Spence, Ranson, and Driver (2000, Experiments 2 and 3), and Spence and Read (2003).

2 . One general concern in comparing performance across experiments is the practical difficulty of gauging participants' true motivation. In a difficult task, motivated participants may perform better than unmotivated participants, but no difference may be observed in less demanding situations. Bearing that in mind, we assume here that there is no special reason to presume that our manipulations systematically induced a lesser degree of enthusiasm in the participants than Driver and Spence's (1994) manipulation did. Therefore, we consider that similar levels of performance are, in principle, a relatively faithful indicator of similar degrees of task difficulty.

3. Initially, we performed a replication of Driver and Spence's (1994) Experiment 2, with the only difference being that the participants made manual responses to the visual targets, as in our previous experiments, whereas the participants in Driver and Spence's (1994) study responded verbally to the appearance of the visual target (and stopped shadowing). In principle, manual responses are more desirable, because they do not require participants to interrupt their shadowing and, therefore, no data have to be discarded. However, after testing 6 participants (see the Appendix), we realized that there was no trace of the synergy effect and, therefore, started our investigation again using verbal responses (see Experiment 3).

4. This was confirmed when the intrusion data were submitted to the arcsine transform and reanalyzed. The new analyses showed that the main effect of side and the interaction between side and visual task were far from significant (both $F \mathrm{~s}<1$ ). The effect of visual task was nearly significant $[F(1,15)=4.4, p=.053]$, with intrusions being slightly more frequent overall during the active task than during the passive task.

5. Indeed, the potential hypothesis that audiovisual links may be observed under phasic attention shifts, but not (or at least, less readily) under sustained attention shifts, is probably an underestimation of the real complexity of the problem. For instance, audiovisual links in exogenous attention shifts (that one may think of as a mainly phasic attention shift) are sometimes not found and appear to depend on the paradigm used (e.g., Ward, McDonald, \& Lin, 2000).

6. One of the reviewers pointed out that another possible factor that may have contributed to the failure to find the expected audiovisual synergy effect in the present experiment is the degree to which the words presented to the attended and the unattended locations were synchronized. In Driver and Spence (1994), the synchrony between the words appearing in each location depended on the ability of the speaker to maintain a constant rate of speech during the recording of the materials. In the present experiment, the words were synthesized, stored in individual computer files, and presented through computerized methods. Presumably, the former study contained more temporal variability between the onsets of the attended and the unattended word lists than the latter did, and this may have caused a more effective capture of attention by the relevant message. There is no way to ascertain these facts, but in any case, this would again be illustrative of the fragility of the audiovisual synergy effect.

\section{APPENDIX \\ Additional Replication of Driver and Spence (1994), Using Simple Manual Responses in the Visual Task}

Every aspect of the method was exactly as in our Experiment 3, except for the number of participants $(n=$ 6; 5 females, all right-handed; mean age $=20$ years, $S D=0.89$ years) and the fact that the visual task required manual responses only (as in Experiments 1 and 2).

The ANOVA on shadowing accuracy included side and visual task as independent variables. The effect of visual task was significant $[F(1,5)=8.1, p<.05]$, with shadowing being more accurate in the passive condition than in the active condition $(56 \%$ vs. $50 \%)$. There was no effect of side $[F<1]$ and no interaction between the two factors $[F(1,5)=1.8, p=.230]$. Overall, intrusions were very infrequent $(M=0.9 \%, S D=$ 0.8 ); however, we ran an ANOVA with the same factors as those in the previous analyses. Neither the main effects nor the interaction was significant (all $p \mathrm{~s}>.25$ ).

(Manuscript received July 21, 2003;

revision accepted for publication June 17, 2004.) 